\title{
RETRIEVAL OF AEROSOL PHASE FUNCTION AND POLARIZED PHASE FUNCTION FROM POLARIZATION OF SKYLIGHT FOR DIFFERENT OBSERVATION GEOMETRIES
}

\author{
L. $\mathrm{Li}^{1}$, L. L. $\mathrm{Qie}^{1}, \mathrm{H} . \mathrm{Xu}^{1}$, Z. Q. $\mathrm{Li}^{1, *}$ \\ ${ }^{1}$ Institute of Remote Sensing and Digital Earth, Chinese Academy of Sciences, Beijing, China - Lizq@ @adi.ac.cn
}

Commission III, WG III/8

KEY WORDS: Aerosol, Polarization, Phase function, Polarized phase function, Retrieval, Sun-sky radiometer

\begin{abstract}
:
The phase function and polarized phase function are important optical parameters, which describe scattering properties of atmospheric aerosol particles. Polarization of skylight induced by the scattering processes is sensitive to the scattering properties of aerosols. The Stokes parameters $I, Q, U$ and the polarized radiance $L_{p}$ of skylight measured by the CIMEL dual-polar sun-sky radiometer CE318DP can be use to retrieve the phase function and polarized phase function, respectively. Two different observation geometries (i.e., the principal plane and almucantar) are preformed by the CE318-DP to detect skylight polarization. Polarization of skylight depends on the illumination and observation geometries. For the same solar zenith angle, retrievals of the phase function and the polarized phase function are still affected by the observation geometry. The performance of the retrieval algorithm for the principal plane and almucantar observation geometries was assessed by the numerical experiments at two typical high and low sun's positions (i.e. solar zenith angles are equal to $45^{\circ}$ and $65^{\circ}$ ). Comparing the results for the principal plane and almucantar geometries, it is recommended to utilize the principal plane observations to retrieve the phase function when the solar zenith angle is small. The Stokes parameter $U$ and the polarized radiance $L_{p}$ from the almucantar observations are suggested to retrieve the polarized phase function, especially for short wavelength channels (e.g., 440 and $500 \mathrm{~nm}$ ).
\end{abstract}

\section{INTRODUCTION}

The scattering and absorption of the electromagnetic radiation by the atmospheric aerosol particles have direct effects on the radiation budget and climate change of the Earth-atmospheric system (Liou, 2002, Wendisch and Yang, 2012). The phase function and polarized phase function are two elements of the scattering phase matrix, which describe scattering properties of aerosol particles (Kokhanovsky, 2003, Wendisch and Yang, 2012). The solar electromagnetic radiation incident at the top of the atmosphere (TOA) is unpolarized. After interacting with suspended particles in the atmosphere, the scattered skylight becomes partially polarized (Smith, 2007). Polarization of skylight is sensitive to scattering properties of atmospheric aerosols, which can be used to retrieve the aerosol phase function and polarized phase function ( $\mathrm{Li}$ et al., 2006).

The CIMEL dual-polar sun-sky radiometer (CE318-DP) is a widely adopted ground-based polarimetric instrument that can measure linear polarization of skylight (e.g., Stokes parameters $I, Q, U$, degree of linear polarization, and polarized radiance) at multiple wavelengths ( $\mathrm{Li}$ et al., 2014). The CE318-DP routinely conducts polarization measurements in the principal plane (i.e., PPP). For this observation geometry, the relative azimuthal angle is fixed at $0^{\circ}$ in the solar direction or $180^{\circ}$ in the anti-solar direction, and the viewing zenith angle is changed in the principal plane (Li et al., 2015). Considering that measurements of $U$ are all close to 0 in the principal plane for different wavelength bands, this information is hard to utilized ( $\mathrm{Li}$ et al., 2014). Then polarization measurements in the almucantar geometry (i.e., ALMP) with viewing zenith angle equal to the solar zenith and varied azimuthal angles in the azimuth plane are additionally conducted

* Corresponding author
(Li et al., 2015). The total radiance $I$ and polarized radiance $L_{p}$ of skylight measured by the CE318-DP can be utilized to retrieve the phase function $\left(P_{11}\right)$ and polarization phase function $\left(P_{12}\right)$, respectively ( $\mathrm{Li}$ et al., 2006). $L_{p}$ is derived from the Stokes parameters $Q$ and $U$. Therefore, $Q$ and $U$ can also be applied to retrieve $P_{12}$ directly.

Polarization of skylight depends on the illumination and observation geometries. For the same illumination geometry, retrievals of the phase function and the polarized phase function are still affected by the observation geometry (e.g., viewing zenith angle, and relative azimuthal angle). The maximum available scattering angles (defined as the angles between the solar incident direction and the viewing directions) in the principal plane and almucantar observation geometries are quite different for the same solar zenith angle (SZA). In this study, retrievals of the phase function and the polarized phase function are performed at two typical high and low sun's positions (i.e. solar zenith angles are equal to $45^{\circ}$ and $65^{\circ}$ ), respectively. The errors of retrievals from the Stokes parameters $I, Q, U$, and polarized radiance $L_{p}$ at different solar zenith angles for the principal plane and almucantar observation geometries are systematically discussed .

\section{METHOD DESCRIPTION}

\subsection{Simulation of skylight polarization for different geome- tries}

Polarization of skylight is traditionally described by the fourdimensional Stokes vector $\vec{S}=(I, Q, U, V)^{\mathrm{T}}$ (superscript $\mathrm{T}$ indicates transpose). The Stokes parameter $I$ is a measure of the total radiance. $Q$ and $U$ describe the linearly polarized radiation. $V$ describes the circularly polarized radiation (Wendisch 
and Yang, 2012). Only the first three Stokes parameters $I, Q$, and $U$ can be detected by the CE318-DP with linear polarizers (Li et al., 2014). Measurements of skylight polarization in different ranges of scattering angles will be obtained at two solar zenith angles (i.e., $\theta_{0}=45^{\circ}, 65^{\circ}$ ) for the principal plane and almucantar observations. The maximum range of scattering angles is $0^{\circ} \leqslant \Theta \leqslant \theta_{0}+90^{\circ}$ for the principal plane observations. Whereas the maximum range is $0^{\circ} \leqslant \Theta \leqslant 2 \theta_{0}$ for the almucantar observations. When the solar zenith angle is equal to $45^{\circ}$, the maximum ranges are $0^{\circ}-135^{\circ}$ for principal plane observations, and $0^{\circ}-90^{\circ}$ for almucantar observations. When the solar zenith angle is equal to $65^{\circ}$, the maximum ranges are $0^{\circ}-155^{\circ}$ and $0^{\circ}-$ $130^{\circ}$, correspondingly. Since the CE318-DP observations are not continuous, the measurements of sky radiance and polarization can only be executed at some fixed angles in the principal and azimuth planes (Li et al., 2015). Therefore, the ranges of scattering angles obtained from actual observations are smaller.

To simulate the linear polarization of skylight measured by the CE318-DP for different observation geometries, a Mie code was used to calculate the single scattering by spherical biomass burning aerosols. Then the optical properties (e.g., extinction coefficient, single scattering albedo and scattering phase matrix) of aerosol particles were further put into the Successive Orders of Scattering (SOS) vector radiative transfer model to calculate the Stokes parameters of skylight in the principal and azimuth planes (Deuze et al., 1989).The input parameters are listed in Table 1. Results of the simulated Stokes parameters $I, Q$, and $U$ are normalized by the extraterrestrial solar irradiance $F_{0}$. Then, the normalized polarized radiance is derived as:

$$
L_{p}=\sqrt{Q_{\mathrm{nor}}^{2}+U_{\mathrm{nor}}^{2}}=\sqrt{\left(\frac{\pi Q}{F_{0}}\right)^{2}+\left(\frac{\pi U}{F_{0}}\right)^{2}} .
$$

\begin{tabular}{|c|c|c|}
\hline \multicolumn{2}{|l|}{ Parameter } & value \\
\hline \multicolumn{2}{|l|}{ Wavelength $(\lambda)$} & $\begin{array}{l}440,500,675,870,1020 \\
1640 \mathrm{~nm}\end{array}$ \\
\hline \multicolumn{2}{|c|}{ Solar zenith angle $\left(\theta_{0}\right)$} & $45^{\circ}, 65^{\circ}$ \\
\hline Scattering & PPP & $\begin{array}{l}1^{\circ}-133^{\circ}\left(\theta_{0}=45^{\circ}\right) \\
1^{\circ}-153^{\circ}\left(\theta_{0}=65^{\circ}\right)\end{array}$ \\
\hline angle $(\Theta)$ & ALMP & $\begin{array}{l}2^{\circ}-90^{\circ}\left(\theta_{0}=45^{\circ}\right) \\
2^{\circ}-130^{\circ}\left(\theta_{0}=65^{\circ}\right)\end{array}$ \\
\hline \multicolumn{2}{|l|}{ Albedo $\left(\rho_{g}\right)$} & $\begin{array}{l}0.03 \text { for } 440 \mathrm{~nm} \\
0.06 \text { for } 500-675 \mathrm{~nm} \\
0.2 \text { for } 870-1640 \mathrm{~nm}\end{array}$ \\
\hline \multicolumn{2}{|c|}{ Particle size distribution } & bimodal log-normal \\
\hline & $\begin{array}{l}r_{\text {mean }, 1} \\
\sigma_{1}\end{array}$ & $\begin{array}{l}82 \mathrm{~nm} \\
0.4\end{array}$ \\
\hline $\begin{array}{l}\text { Aerosol model: } \\
\text { biomass } \\
\text { burning }\end{array}$ & $\begin{array}{l}r_{\text {mean }, 2} \\
\sigma_{2} \\
C_{1} / C_{2} \\
m \\
\text { shape }\end{array}$ & $\begin{array}{l}1520 \mathrm{~nm} \\
0.6 \\
65700 \\
1.52-\mathrm{i} 0.025 \\
\text { sphere }\end{array}$ \\
\hline \multicolumn{2}{|c|}{ Aerosol optical depth $(\tau)$} & $0.5(440 \mathrm{~nm})$ \\
\hline
\end{tabular}

Table 1. Input parameters for simulating of polarization measurements of the polarized sun-sky radiometer

\subsection{Retrieval of phase function and polarized phase func- tion}

For a group of spherical particles, the single scattering property is described by the scattering phase matrix with only four independent elements (Liou, 2002):

$$
\mathbf{P}=\left[\begin{array}{cccc}
P_{11} & P_{12} & 0 & 0 \\
P_{12} & P_{11} & 0 & 0 \\
0 & 0 & P_{33} & P_{34} \\
0 & 0 & -P_{34} & P_{33}
\end{array}\right]
$$

For the unpolarized incident light, only two elements of the scattering phase matrix (i.e., $P_{11}$ and $P_{12}$ ) can be retrieved from the measurements of linear polarization of skylight. The polarized phase function is often expressed as the normalized form as $P_{12} / P_{11}$. The phase function and polarized phase function are retrieved from the normalized radiance $I_{\text {nor }}$ and the normalized polarized radiance $L_{p}$ in the form (Devaux et al., 1998, Vermeulen et al., 2000, Li et al., 2006)

$$
\begin{gathered}
P_{\mathrm{a}, 11}=\frac{I_{\mathrm{nor}}-\left[I_{\mathrm{nor}}^{*}\left(\omega_{0}\right)-I_{\mathrm{nor}, 0}^{*}\left(\omega_{0}\right)\right]}{I_{\text {nor }, 0}^{*}\left(\omega_{0}\right)} P_{\mathrm{a}, 11}^{*} \\
+\frac{I_{\mathrm{nor}}-I_{\text {nor }}^{*}\left(\omega_{0}\right)}{I_{\text {nor }, 0}^{*}\left(\omega_{0}\right)} \frac{\tau_{\mathrm{m}}}{\omega_{0} \tau_{\mathrm{a}}} P_{\mathrm{m}, 11}, \\
P_{\mathrm{a}, 12}=\frac{L_{\mathrm{p}}}{L_{\mathrm{p}}^{*}\left(\omega_{0}\right)} P_{\mathrm{a}, 12}^{*}+\frac{L_{\mathrm{p}}-L_{\mathrm{p}}^{*}\left(\omega_{0}\right)}{L_{\mathrm{p}}^{*}\left(\omega_{0}\right)} \frac{\tau_{\mathrm{m}}}{\omega_{0} \tau_{\mathrm{a}}} P_{\mathrm{m}, 12},
\end{gathered}
$$

where $\quad P_{\mathrm{a}, 11}=$ aerosol phase function

$P_{\mathrm{a}, 12}=$ aerosol polarized phase function

$P_{\mathrm{a}, 11}^{*}=$ initial aerosol phase function

$P_{\mathrm{a}, 12}^{*}=$ initial aerosol polarized phase function

$\tau_{\mathrm{a}}=$ aerosol optical thickness

$\omega_{0}=$ aerosol single scattering albedo

$P_{\mathrm{m}, 11}=$ molecular phase function

$P_{\mathrm{m}, 12}=$ molecular polarized phase function

$\tau_{\mathrm{m}}=$ molecular optical thickness

$I_{\text {nor }}^{*}$ and $L_{\mathrm{p}}^{*}$ are the normalized radiance and polarized radiance simulated with initial guessed aerosol properties (e.g., Junge size distribution and the refractive index $m=1.4$ ), and $I_{\text {nor }, 0}^{*}$ is the corresponding parameter calculated with black surface (Li et al., 2006). Similarly, the polarized phase function can be deduced from the Stokes parameters $Q$ and $U$ as

$$
\begin{aligned}
& P_{\mathrm{a}, 12}=\frac{Q_{\mathrm{nor}}}{Q_{\text {nor }}^{*}\left(\omega_{0}\right)} P_{\mathrm{a}, 12}^{*}+\frac{Q_{\mathrm{nor}}-Q_{\text {nor }}^{*}\left(\omega_{0}\right)}{Q_{\text {nor }}^{*}\left(\omega_{0}\right)} \frac{\tau_{\mathrm{m}}}{\omega_{0} \tau_{\mathrm{a}}} P_{\mathrm{m}, 12}, \\
& P_{\mathrm{a}, 12}=\frac{U_{\mathrm{nor}}}{U_{\text {nor }}^{*}\left(\omega_{0}\right)} P_{\mathrm{a}, 12}^{*}+\frac{U_{\mathrm{nor}}-U_{\text {nor }}^{*}\left(\omega_{0}\right)}{U_{\text {nor }}^{*}\left(\omega_{0}\right)} \frac{\tau_{\mathrm{m}}}{\omega_{0} \tau_{\mathrm{a}}} P_{\mathrm{m}, 12} .
\end{aligned}
$$

$Q_{\text {nor }}^{*}$ and $U_{\text {nor }}^{*}$ are also simulated with the initial guessed aerosol properties.

\section{RESULTS AND DISCUSSION}

The performance of the retrieval algorithm for different observation geometries was assessed. The Stokes parameter $I$ simulated with the biomass burning aerosol model was applied to retrieve the aerosol phase function $P_{11}$, and the corresponding Stokes parameters $Q, U$, and the polarized radiance $L_{p}$ were used to retrieve the normalized polarized phase function $-P_{12} / P_{11}$. The true values of $P_{11}$ and $-P_{12} / P_{11}$ obtained in the single scattering process were adopted to evaluate the retrievals. 


\subsection{Results for the Principle Plane Geometry}

Figure1 gives the results of $P_{11}$ retrieved from the Stokes parameter $I$ at solar zenith angles of $45^{\circ}$ and $65^{\circ}$ for the CE318-DP principal plane observation geometry. Combined the sky radiance observations with polarization observations, the maximum scattering angles can reach up to $133^{\circ}$ for SZA of $45^{\circ}$ and $153^{\circ}$ for SZA of $65^{\circ}$, respectively. From Figure 1, the inversion results in the 675 to $1640 \mathrm{~nm}$ channels are in good agreement with the true values in both illumination geometries. The errors mainly occur around the forward direction at the scattering angles within $10^{\circ}$. Except for those scattering angles, the errors on other scattering angles are basically within 5\%. The longer the wavelength is, the smaller the error is. However, the relative errors in the channels centered at 440 and $500 \mathrm{~nm}$ are obvious. Large errors mainly occur when the scattering angle is greater than $90^{\circ}$. This is in consistent with the previous results that the inversions of the phase function in the principal plane are good in the channels of $675-1640 \mathrm{~nm}$, while the errors at short wavelengths are more obvious (Li et al., 2006). Comparing the results of $P_{11}$ retrieved from the principal plane observations at two solar zenith angles, it can be seen that more results of $P_{11}$ are obtained from the observations with SZA of $65^{\circ}$ than those with SZA of $45^{\circ}$. However, when SZA is equal to $65^{\circ}$, the relative errors at large scattering angles are larger than which of SZA equal to $45^{\circ}$. The shorter the wavelength is, the more obvious the relative errors are at large scattering angles. In general, the differences in results at two solar zenith angles are not significant in the channels of 675 $-1640 \mathrm{~nm}$. They are all close to the true values.

Only Stokes parameter $Q$ was adopted to retrieve the polarized phase function $-P_{12} / P_{11}$ in Figure 2 since $U$ are all close to 0 for different wavelengths in the solar principal plane (Li et al., 2014). Similar to the situation of $P_{11}$, the inversion results of $-P_{12} / P_{11}$ are in good agreement with the true values at the wavelengths of $675,870,1020$, and $1640 \mathrm{~nm}$. However, the results retrieved from the observations at SZA of $45^{\circ}$ and $65^{\circ}$ all deviate explicitly from the true values in the channels of 440 and $500 \mathrm{~nm}$. Outliers can be observed near the scattering angle of $10^{\circ}$ in the results both at SZA of $45^{\circ}$ and $65^{\circ}$. It is mainly due to the fact that the curves of the Stokes parameter $Q$ changing from positive to negative around this angle.

\subsection{Results for the Almucantar Geometry}

The phase function $P_{11}$ retrieved from the Stokes parameter $I$ in the azimuth plane is shown in Figure 3. For the almucantar observations, the maximum scattering angles are only $90^{\circ}$ with SZA of $45^{\circ}$, and $130^{\circ}$ with SZA equal to $65^{\circ}$. Similar to the situation in the principal plane, the relative errors of $P_{11}$ are larger in the shortwave channels (440 and $500 \mathrm{~nm}$ ) than which in the channels of long wavelengths $(675-1640 \mathrm{~nm})$. Relative errors of $P_{11}$ for SZA of $65^{\circ}$ are obviously smaller than which for SZA equal to $45^{\circ}$, expect for the 440 and $1020 \mathrm{~nm}$ channels.

Figure 4 gives the results of the polarized phase function $-P_{12} / P_{11}$ retrieved from the polarization measurements of the almucantar geometry. The Stokes parameters $Q, U$ and the polarization radiance $L_{p}$ are all adopted to retrieve $-P_{12} / P_{11}$. It is obvious that the inversions are closer to corresponding true values when SZA is equal to $65^{\circ}$ in comparison with the case of SZA equal to $45^{\circ}$. When SZA is equal to $45^{\circ}$, the results of $-P_{12} / P_{11}$ deviate from the true values at large scattering angles (i.e., around scattering angles of $80^{\circ}-90^{\circ}$ ). It is also clear that the results derived from $Q, U$ and $L_{p}$ are consistent with each other at most of the scattering angles. Nevertheless, significant outliers appear around the scattering angle of $70^{\circ}$ in the results of $-P_{12} / P_{11}$ derived from $Q$ when SZA is equal to $45^{\circ}$. Likewise,outliers exist around the scattering angle of $115^{\circ}$ when SZA is equal to $65^{\circ}$. It is because the values of $Q$ are close to 0 at these angles. But for the retrievals by $U$ and $L_{p}$, there are no outlier. So, it is better to apply $U$ or $L_{p}$ for the retrieval in the azimuth plane.

Comparing the results for the principal plane and almucantar geometries, it is evident that the results of $P_{11}$ retrieved from the principal plane observations agree better with the true values than the corresponding results retrieved from the almucantar observations when the solar zenith angle is equal to $45^{\circ}$. But the results of $P_{11}$ in the azimuth plane are significant improved when the solar zenith angle expands to $65^{\circ}$. When the solar zenith angle is small (e.g., $45^{\circ}$ ), results at larger ranges of scattering angles can be obtained from the principal plane observations than those retrieved from the almucantar observations. Moreover, there are no obvious difference in the results of $P_{11}$ between SZA of $45^{\circ}$ and $65^{\circ}$ for the 675 to $1640 \mathrm{~nm}$ channels in the principal plane. Thus, it is recommended to utilize the principal plane observations to retrieve the phase function when the solar zenith angle is small. For $-P_{12} / P_{11}$, results retrieved from the principal plane and almucantar observations at 675 to $1640 \mathrm{~nm}$ are all consistent with the true values. Nevertheless, it is also obvious that $-P_{12} / P_{11}$ retrieved from the almucantar observations are better agree with the true values in the channels of 440 and $500 \mathrm{~nm}$ than which in the principal plane. So, it is suggested to use the Stokes parameter $U$ or the polarized radiance $L_{p}$ from the almucantar observations to retrieve the polarized phase function at short wavelengths (such as 440 and $500 \mathrm{~nm}$ ). For long wavelengths (e.g., $675-1640 \mathrm{~nm}$ ), the results of $-P_{12} / P_{11}$ retrieved from the principal plane and almucantar observations are all acceptable.

\section{CONCLUSIONS}

The Stokes parameters $I, Q, U$ and the polarized radiance $L_{p}$ measured by the CIMEL dual-polar sun-sky radiometer (CE318DP) were used for the retrievals of the phase function and the polarized phase function of atmospheric aerosol particles. The performance of the retrieval algorithm for the principal plane and almucantar observation geometries was assessed by the numerical experiments in this study. For the results of $P_{11}$, the relative errors are generally smaller for the principal plane geometry than which for the almucantar geometry, except for the 440 and 500 $\mathrm{nm}$ channels. Outliers exist in the results of $-P_{12} / P_{11}$ around the scattering angles of $10^{\circ}$, and $70^{\circ}$ or $115^{\circ}$ retrieved from the Stokes parameter $Q$ in the principal and azimuth planes, respectively. Comparing the results of $-P_{12} / P_{11}$ in the principal plane and almucantar geometries, it is recommended to adopt the Stokes parameter $U$ or the polarized radiance $L_{p}$ in the almucantar plane for the retrieval, especially in short wavelength channels of 440 and $500 \mathrm{~nm}$

\section{ACKNOWLEDGEMENTS}

This research was supported by National Natural Science Foundation of China (NSFC) (41501390, 41671364).

\section{REFERENCES}

Deuze, J. L., Herman, M. and Santer, R., 1989. Fourier series expansion of the transfer equation in the atmosphere-ocean system. Journal of Quantitative Spectroscopy \& Radiative Transfer 41(6), pp. 483-494. 

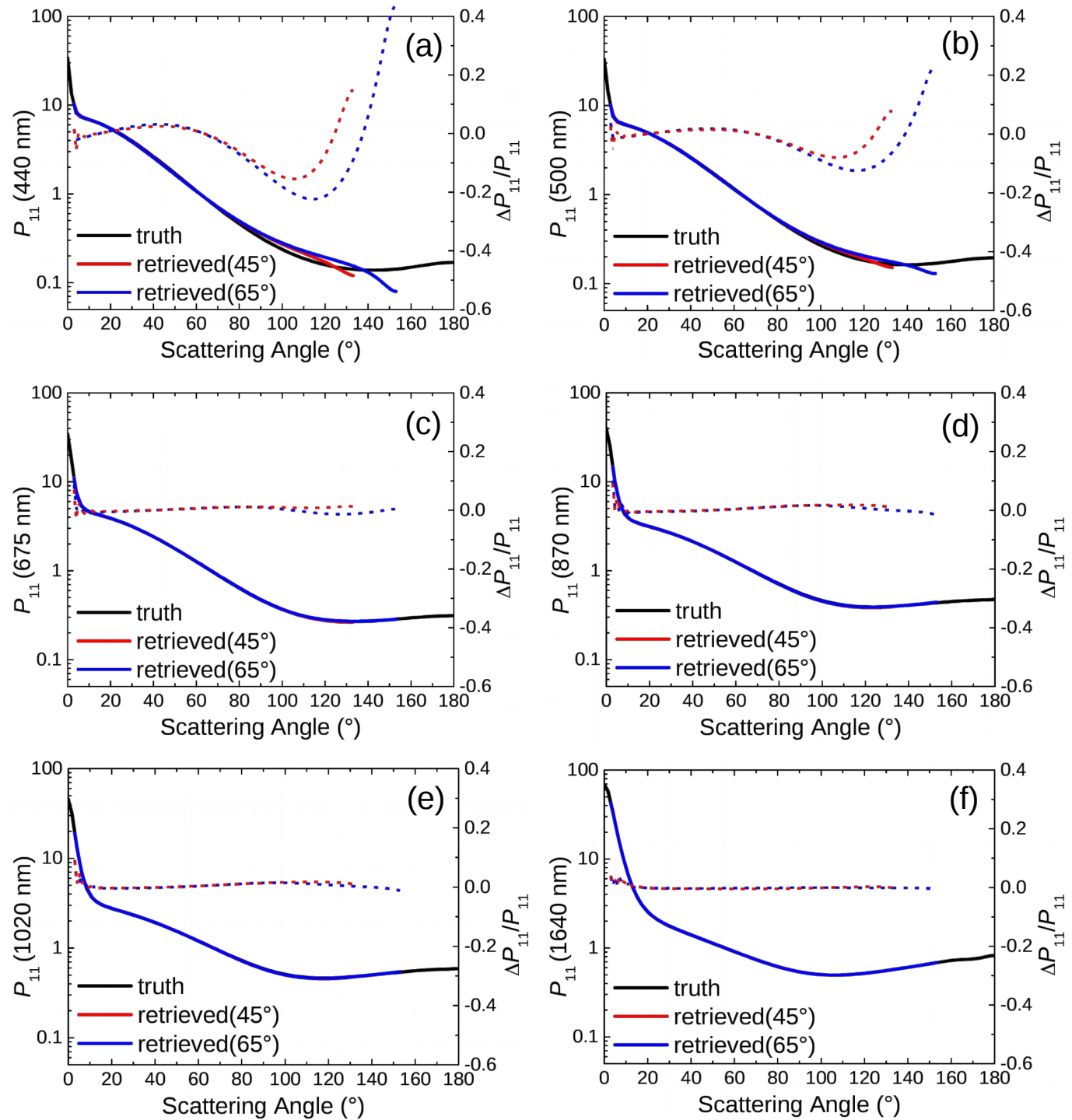

Figure 1. Comparison of $P_{11}$ retrieved from the Stokes parameter $I$ at different solar zenith angles for the principal plane observation geometry. The curve notation is as: red solid lines for results of $P_{11}$ with solar zenith angle (SZA) of $45^{\circ}$; red dashed lines for relative errors with SZA of $45^{\circ}$; blue solid lines for results of $P_{11}$ with SZA of $65^{\circ}$; blue dashed lines for relative errors with SZA of $65^{\circ}$.

Devaux, C., Vermeulen, A., Deuz, J. L., Dubuisson, P., Herman, M., Santer, R. and Verbrugghe, M., 1998. Retrieval of aerosol singlescattering albedo from groundbased measurements: Application to observational data. Journal of Geophysical Research Atmospheres 103(D8), pp. 8753-8761.

Kokhanovsky, A. A., 2003. Polarization Optics of Random Media. Springer, Berlin.

Li, L., Li, Z., Li, K., Blarel, L. and Wendisch, M., 2014. A method to calculate stokes parameters and angle of polarization of skylight from polarized cimel sun/sky radiometers. Journal of Quantitative Spectroscopy \& Radiative Transfer 149(6), pp. 334346.

Li, Z., Goloub, P., Devaux, C., Gu, X., Deuz, J. L., Qiao, Y. and Zhao, F., 2006. Retrieval of aerosol optical and physical properties from ground-based spectral, multi-angular, and polarized sun-photometer measurements. Remote Sensing of Environment 101(4), pp. 519-533.

Li, Z., Li, D., Li, K., Xu, H., Chen, X., Chen, C., Xie, Y., Li, L., Li, L. and Li, W., 2015. Sun-sky radiometer observation net- work with the extension of multi-wavelength polarization measurements. Journal of Remote Sensing 19(3), pp. 495-519.

Liou, K. N., 2002. An Introduction to Atmospheric Radiation. ACADEMIC PRESS.

Smith, G. S., 2007. The polarization of skylight: An example from nature. American Journal of Physics 75(1), pp. 25-35.

Vermeulen, A., Devaux, C. and Herman, M., 2000. Retrieval of the scattering and microphysical properties of aerosols from ground-based optical measurements including polarization. i. method. Appl Opt 39(33), pp. 6207-20.

Wendisch, M. and Yang, P., 2012. Theory of atmospheric radiative transfer : a comprehensive introduction. Wiley- $\mathrm{VCH}$,.

Revised March 2018 

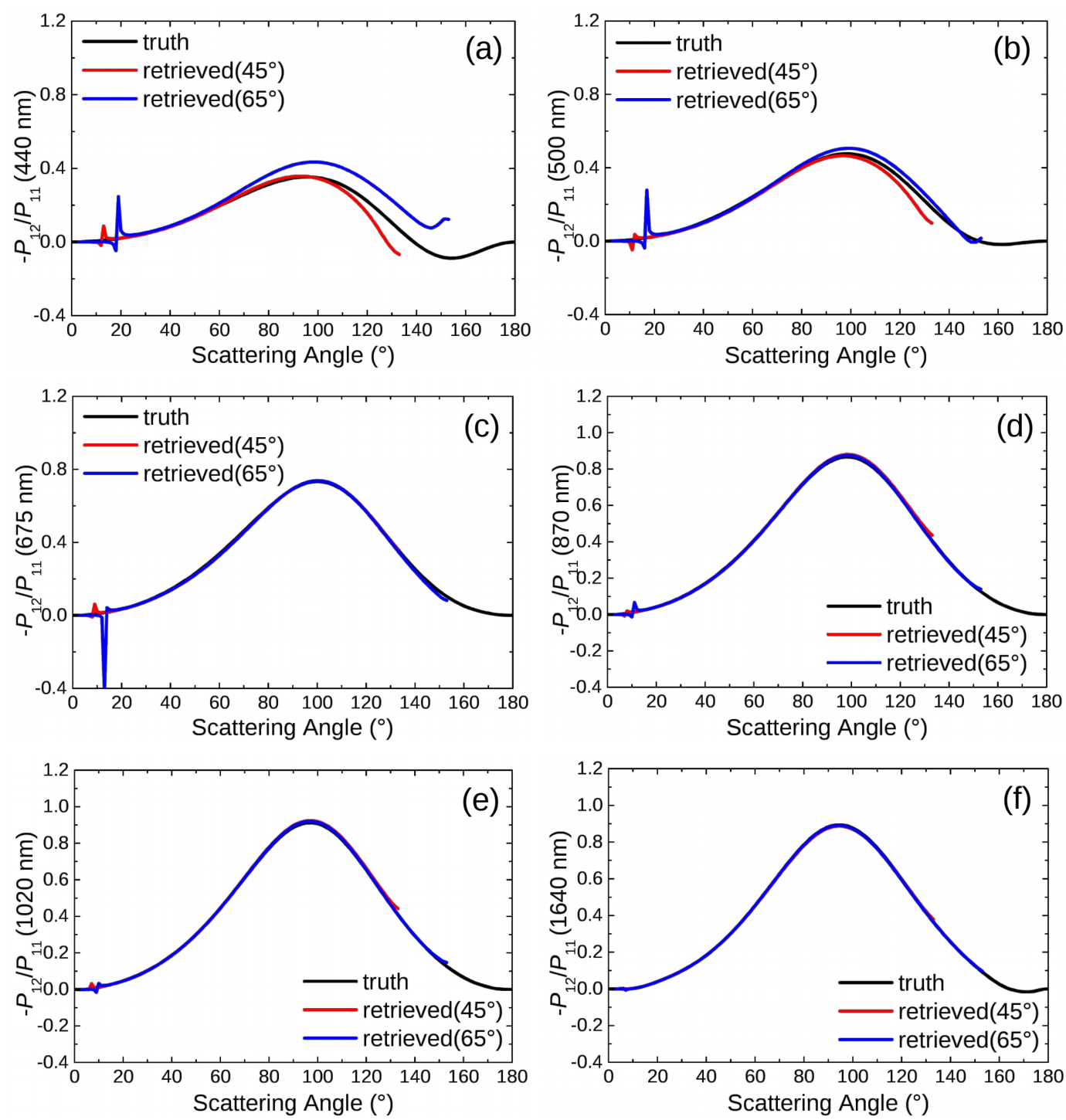

Figure 2. Comparison of $-P_{12} / P_{11}$ retrieved from the Stokes parameter $Q$ at different solar zenith angles for the principal plane observation geometry. The curve notation is as: red solid lines for results with SZA of $45^{\circ}$; blue solid lines for results with SZA of $65^{\circ}$. 

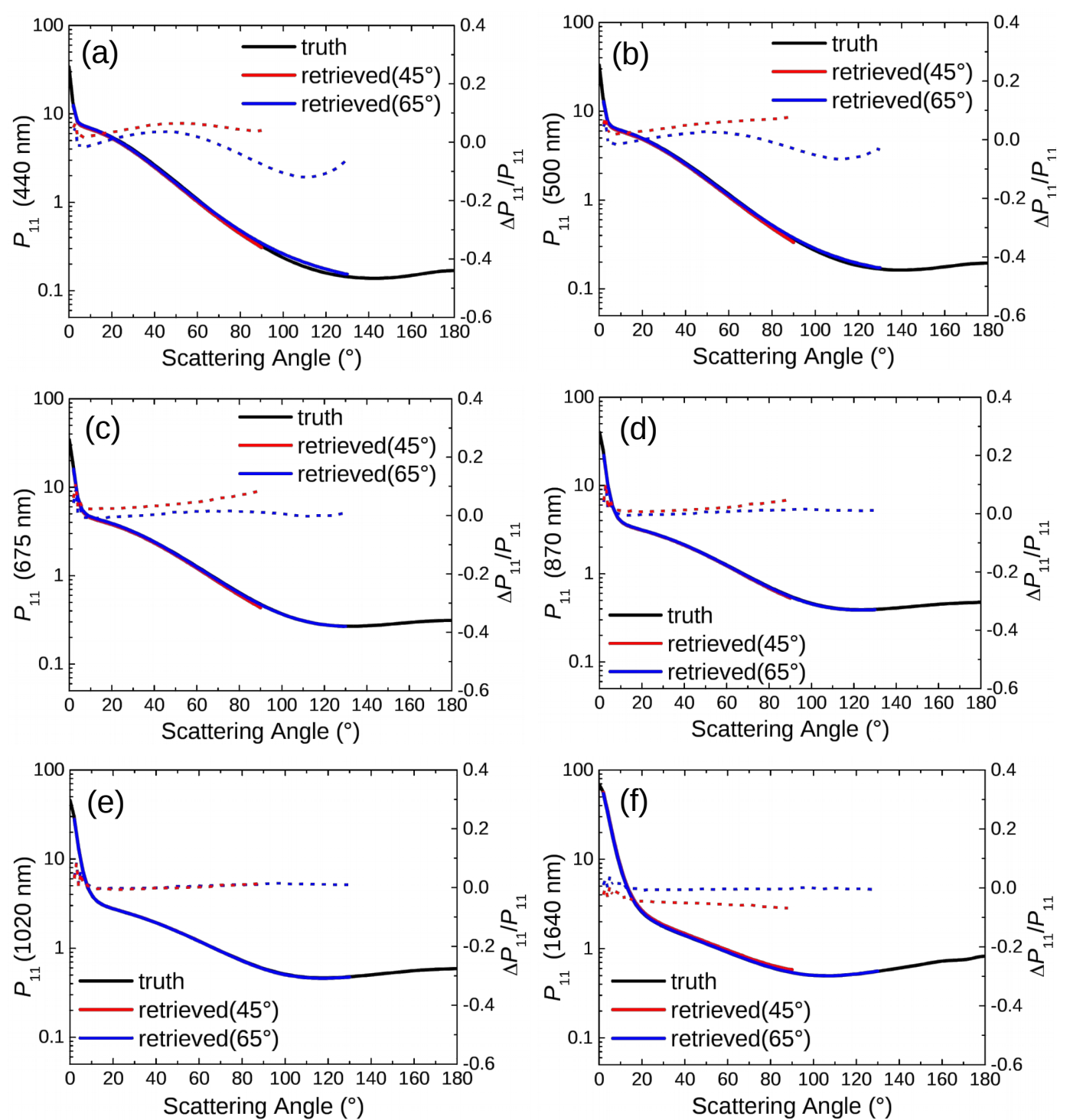

Figure 3. Comparison of $P_{11}$ retrieved from the Stokes parameter $I$ at different solar zenith angles for the almucantar observation geometry. The curve notation is as: red solid lines for results of $P_{11}$ with SZA of $45^{\circ}$; red dashed lines for relative errors with SZA of $45^{\circ}$; blue solid lines for results of $P_{11}$ with SZA of $65^{\circ}$; blue dashed lines for relative errors with SZA of $65^{\circ}$. 

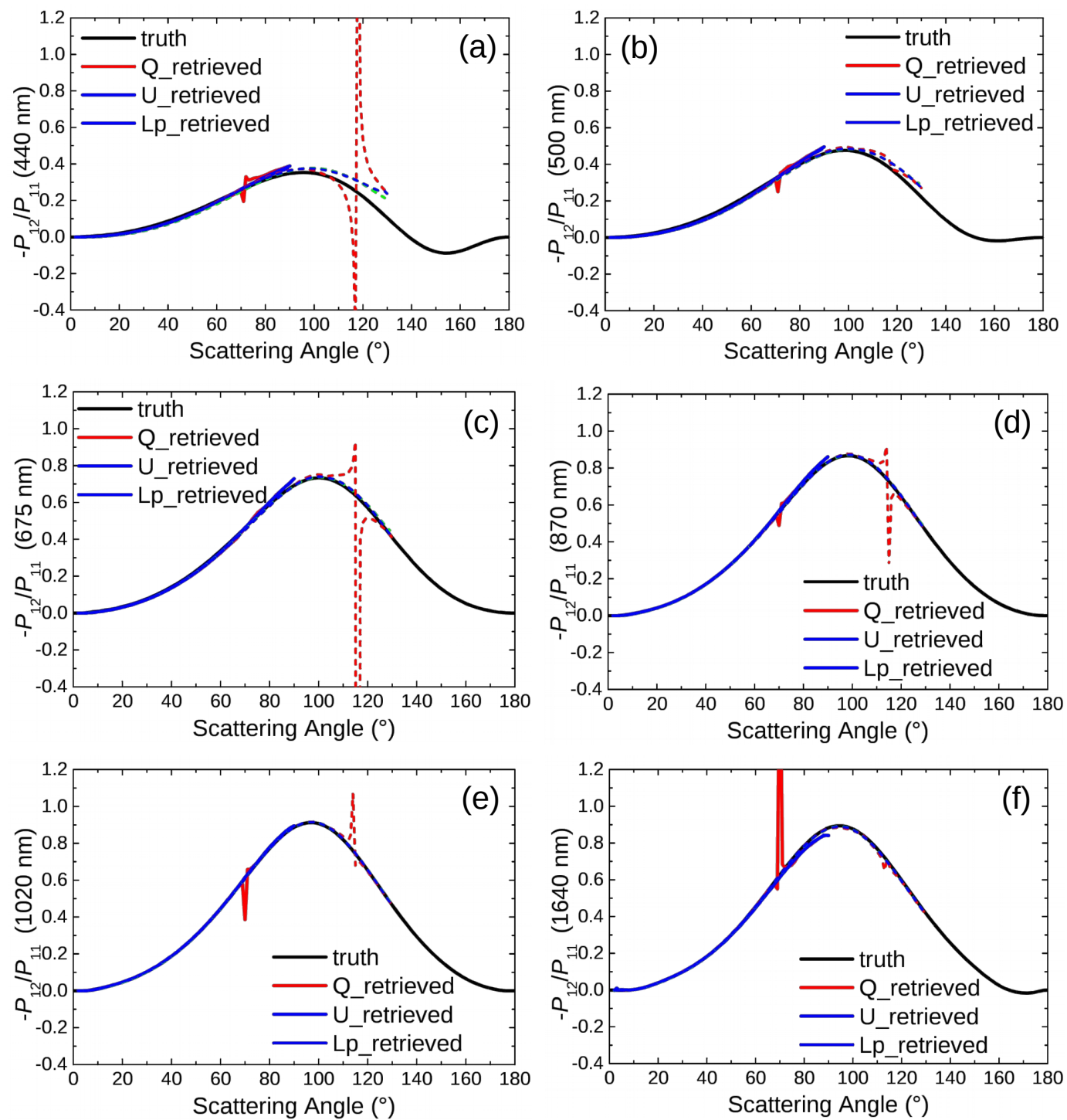

Figure 4. Comparison of $-P_{12} / P_{11}$ retrieved from the Stokes parameters $Q, U$ and the polarized radiance $L_{p}$ at different solar zenith angles for the almucantar observation geometry. The curve notation is as: solid lines for results with SZA of $45^{\circ}$; dashed lines for results with SZA of $65^{\circ}$; red lines for the results derived from $Q$; green lines for the results derived from $U$; and blue lines for the results derived from $L_{\mathrm{p}}$. 